1 Running Head: AETIOLOGICAL ARCHITECTURE OF DEPRESSIVE SYMPTOMS

2

Phenotypic and aetiological architecture of depressive symptoms in a Japanese twin

Yusuke Takahashi ${ }^{1,2}$, Jean-Baptiste Pingault ${ }^{2,3}$, Shinji Yamagata ${ }^{4}$, Juko Ando ${ }^{5}$

1: The Hakubi Center for Advanced Research and Graduate School of Education, Kyoto

9 University, Kyoto, Japan; 2: Department of Clinical, Educational and Health Psychology,

10 Division of Psychology and Language Sciences, University College London, United

11 Kingdom; 3: Social, Genetic \& Developmental Psychiatry Centre, King’s College London,

12 United Kingdom; 4: Graduate School of Education and Human Development, Nagoya

13 University, Aichi, Japan; 5: Faculty of Letters, Keio University, Tokyo, Japan

15 Word count of the main body text: 4,119 words

17 Correspondence concerning this article should be addressed to Yusuke Takahashi, The

18 Hakubi Center for Advanced Research and Graduate School of Education, Kyoto

19 University, Yoshida Honmachi, Sakyo-ku, Kyoto, 606-8501, Japan. Email:

takahashi.yusuke.3n@kyoto-u.ac.jp 
Abstract

2 Background: The phenotypic and aetiological architecture of depression symptomatology has been mostly studied in Western samples. In this study, we conducted a genetically informed factor analysis to elucidate both the phenotypic and aetiological architectures of self-reported depression among a Japanese adult twin sample. Methods: Depressive symptoms assessed by Zung's Self-rating Depression Scale were self-rated by 425 twin pairs (301 monozygotic and 124 dizygotic twin pairs) in a community sample in Japan.

8 Results: An exploratory factor analysis extracted three symptom domains representing

9 cognitive, affective, and somatic symptomatology. A confirmatory factor analysis demonstrated that a bi-factor solution fitted better than the alternative solutions, implying that depression may be defined as a combination of a single general construct and three factors specific to each of the three symptom domains. A multivariate genetic analysis with the bi-factor solution showed that the general factor was substantially heritable (47\%), and that only the affective symptom domain was significantly heritable (29\%) among the three specific factors, their remaining variance being explained by non-shared environmental influences. Conclusions: Depression symptomatology appears to be adequately captured by a substantially heritable general factor. The heritability of this factor (47\%) in a Japanese adult sample is in line with commonly reported heritability estimates for depression. The three specific factors - cognitive, affective, and somatic - are mostly explained by non-shared environmental factors, which include measurement error. The 
1 extent to which these specific factors are uniquely associated with correlates of depression

2 when the general factor is accounted for should be investigated in future studies.

4 Keywords: depression, heritability, behavioural genetics, twin study, bi-factor model 


\section{Phenotypic and aetiological architecture of depressive symptoms in a Japanese twin}

\section{sample}

According to the report by the World Health Organisation (2017), more than 300

million people suffer from depression across the globe and depression is ranked in the top-ten causes of early death. In Japan, the lifetime prevalence of depression is comprised between $3 \%$ and $7 \%$ (i.e. major depressive disorder as defined by the DSM-5 criteria; American Psychiatric Association, 2013, Kawakami, 2007, Kessler \& Bromet, 2013).

Although the prevalence is somewhat lower in Japan than in Western countries (Kessler \& Bromet, 2013), major depression still constitutes a rather common disorder compared with other psychiatric disorders.

Accurately assessing depressive symptoms within and across populations constitutes an essential first step before elucidating the aetiology of depression. However, differences in the phenotypic architecture of dimensional measures of depression are found across populations. The typical three factor structure (i.e. cognitive, affective, and somatic symptom domains) of Zung's (1965) Self-rating Depression Scale or SDS is sometimes found to differ across ethnically different samples. For example, four factors were found in Spanish and US samples (Passik et al., 2000; Romera et al., 2008). Within the Spanish sample the symptom factors reflected core depressive, cognitive, anxiety, and somatic factors; whereas in the US sample, the factors were slightly different, reflecting cognitive 
1 symptoms, manifested by depressed mood, somatic non-eating, and somatic eating

2 behaviour. Meanwhile, fewer factors tend to be extracted within Asian samples. For example, an early study of ethnically Chinese people drawn from community-based clinical and non-clinical samples from Hong Kong found two factors described as: depression with somatic complaints and negative view of life (Lee et al., 1994). A study of the Japanese general population found two factors: depressed affect and emptiness (Chida et al., 2004).

7 However, three studies in Japanese undergraduates or mothers around birth have replicated

8 the original three factors: cognitive, affective, and somatic symptoms (Kitamura et al.,

9 2004; Sakamoto et al., 1998; Sugawara et al., 1999). Kitamura et al. (2004) used the largest sample and Sakamoto et al. (1998) found the same structure using confirmatory factor analytic techniques. The content captured by the factors reported in these studies, in particular those reporting a three-factor solution, relate to the content of diagnostic criteria of the DSM-5. For example, the cognitive symptom domain reflects the DSM-5 diagnostic criteria such as diminished ability to think or concentrate, or indecisiveness, and recurrent thoughts of death; the affective symptom domain reflects a depressed mood, feelings of worthlessness or excessive or inappropriate guilt and the somatic symptom domain reflects significant weight loss or gain, and insomnia or hypersomnia. the phenotypical structure of depression using exploratory and confirmatory factor analyses. 
1 factor. Additionally, we tested alternative factor solutions, including higher-order factor and

2 bi-factor models, which both comprise general and specific factors and offer more

3 parsimonious representations of the relationships between the multiple symptom domains

4 of depression. Especially, a bi-factor analysis allows us (a) to simultaneously model

5 depressive symptomatology at the general and the domain-specific levels, (b) to examine

6 whether domain-specific factors retain any unique variance, unshared with other domains

7 (e.g. Holzinger \& Swineford, 1937).

Investigating genetic and environmental influences in depression is important

9 because it can provide a modelling scheme that represents the complex and multi-factorial nature of symptom domain risks (Byers et al., 2009). A review of twin research prior to 1999 from Australian, Swedish, British, and American samples by Sullivan et al. (2000) showed that depression has a heritable component that does not appreciably differ between community and clinical samples $\left(h^{2}=37 \%\right.$ [95\%CI: $\left.28 \%, 42 \%\right]$ for community samples, and $h^{2}=43 \%$ [95\%CI: 21\%, 58\%] for clinical samples). More recently, a study with a large sample of Scottish families demonstrated that the heritability of depression was between $28 \%$ to $44 \%$ (Fernandez-Pujals et al., 2015), whereas in the US sample with an extended

17 family design the heritability estimate of depressive symptoms was not significant $\left(h^{2}=\right.$ 8.6\% [95\%CI: 0\%, 57\%, Byers et al., 2009). For non-Western countries, previous research

19 found that the heritability of depression was also moderate, specifically ranging from $34 \%$ 
1 al., 2009; Chen et al., 2014; Chen \& Yu, 2015). However, interestingly, although factor

2 analyses at the phenotypic level pointed toward the existence of multiple factors, almost all

3 prior research on the aetiology of depression was based on the total score only, except for a

4 few studies (e.g. McGue \& Christensen, 1997). Therefore, few behavioural genetic studies

5 on depression have taken into consideration the multi-dimensionality of depressive

6 symptoms.

In this study, we aimed to examine the aetiological architecture of depressive

8 symptoms based on findings from phenotypic confirmatory factor analyses. More

9 specifically, the purpose of the present paper was three-fold. First, we examined the

10 factorial structure of the SDS in a twin sample from a Japanese community sample to

11 elucidate the phenotypic structure of depressive symptoms. We compared three

12 hypothetical models (i.e. multiple-factor, higher-order factor, and bi-factor models; see Fig.

13 1). Second, we estimated the relative influence of genetic and environmental influences on

14 each domain of depression symptomatology assessed by the SDS. Third, we investigated

15 the relationships between genetic and environmental influences underlying the depressive

symptom domains to determine the role these factors play in their observed covariance.

\section{Method}

Subjects and Questionnaires

A mail survey was conducted as part of the ongoing longitudinal Keio Twin Study 
1 total number of participants was 425 twin pairs including $301 \mathrm{MZ}$ twin pairs (211 female

$2 \mathrm{MZ}, 87$ male MZ, and 3 sex-unknown twin pairs) and $124 \mathrm{DZ}$ twin pairs (56 female DZ, 26

3 male DZ, and 42 opposite-sex twin pairs). The twins were 23.41 years old $(S D=4.24)$. The

4 participants completed the Japanese version of the SDS (20 items, 4-point Likert scale;

$5 \quad$ Fukuda \& Kobayashi, 1983; Zung, 1965) which is widely and commonly used to assess

6 individual differences in depressive symptoms, adequately capturing multiple symptom

7 domains (Cheung \& Power, 2012). The descriptive statistics are shown in Table 1. As a

8 whole, the scale was adequately internally consistent $(\omega=.89)$. The zygosity was

9 determined by questionnaire items on the physical similarity and confusion of the twins by

10 family members, friends and strangers (Ooki et al., 1990; Torgersen, 1979), and has been

11 shown to categorise twin pairs correctly in over 93\% of cases (Magnus et al., 1983; Ando et

12 al., 2013).

Twin Methods

Heritability, or the proportion of the observed variability on a measure directly

attributable to genetic influences is estimated by comparing within-pair similarity (such as

with Pearson's $r$ ) in MZ twins who share $100 \%$ of their genetic material to within-pair similarity in DZ twins who share on average $50 \%$ of their segregated genes (Knopik et al., 2016). Using structural equation modelling, the phenotypic variance can be decomposed into four components: additive genetic (A), non-additive genetic (D), shared environmental 
1 genetic effects on the phenotype of interest. D represents the interactive contribution of

2 alleles within a single locus; $\mathrm{C}$, or shared environment, estimates environmental influences

3 that make twins similar, regardless of their zygosity; and E, or non-shared environment,

4 represents environmental influences that make twins different, and also includes

5 measurement error. To reveal which influences significantly contribute to phenotypic

6 variances, five models were systematically compared. We fitted the ADE model, ACE

7 model, AE model, CE model, and E model and evaluated the relative explanatory power of

8 each model. Furthermore, to investigate the nature of the relationships between the multiple

9 sets of symptom domains, we used multivariate genetic analyses to parameterise the

10 variance and covariance between multiple symptoms at the same time. All the behavioural

11 genetic analyses were performed on the raw data using the full information maximum

12 likelihood estimation implemented in the R packages OpenMx 2.0 (Neale et al., 2016) and

13 lavaan (Rosseel, 2012). The model fits were evaluated by using the chi-square index and

14 Akaike's Information Criterion (AIC; Akaike, 1987) which is calculated by $\chi^{2}-2 d f$ as well

15 as the Bayesian Information Criterion (BIC, Schwarz, 1978; Sclove, 1987). The model with the lowest AIC and/or BIC indicates the best fit.

Descriptive Statistics and Exploratory and Confirmatory Factor Analyses 
1 correlation between age and the total SDS score $(r=-.05, p=.18)$, and no significant

2 differences between the sexes $(t(837)=1.09, p=.28)$. Although the effects of age and sex on depressive symptoms were statistically insignificant, the regression residuals obtained

4 after controlling for these two factors were used in all of the following analyses (McGue \& Bouchard, 1984).

The SDS items were subjected to maximum likelihood factor analysis with direct

Oblimin rotation, using one twin randomly selected from each twin pair. Five items (i.e.

[a]: I have trouble with constipation, [b]: Morning is when I feel the best [reversed item], [c]: I feel that others would be better off if I were dead, [d]: I find it easy to do the things I used to [reversed item], and [e]: I notice that I am losing weight) showed low factor loadings, which were less than .35 on all components and showed high complexity over 2.0, which means none of these five items could not contribute efficiently to a simple structure (Hofmann, 1978). A previous study also found that the first two items (i.e. [a] and [b]) had low loadings when a principal component analysis was conducted (Sakamoto et al., 1998). Item [c] should be classified in the affective symptom domain. However, in line with the previous study (Sakamoto et al., 1998), this was not loaded on any factors, maybe because the item exhibited a floor effect among the non-clinical samples (over two thirds of the sample answered "little or none of the time [1]" to this item) and the correlation between item [c] and other items became obscured. The content of item [d] is somewhat unspecific and hard to allocate to a specific symptom domain. Although item [e] can clearly be 
1 included in the somatic symptom domain (American Psychiatric Association, 2013), in this

2 sample, it was dropped as loading was $<.35$ and complexity $>2.0$. Therefore, these five

3 items were removed from all subsequent analyses.

Our examination of a scree plot obtained with simulations implemented in the R package psy (Falissard, 2012) suggested three factors (Table 1 and Fig. 2). We next performed a confirmatory factor analysis using the R package lavaan (Rosseel, 2012). In all tested models, the factor loadings were equally constrained across twins and zygosity groups for each item of the depression scale, so that the same factor model was specified

9 across twins in a pair and across zygosity groups (e.g. Olsen \& Kenny, 2006; Wood et al., 2008). The three-factor model fitted reasonably well to the data, compared to other factor models. The first factor was defined by five items and could be interpreted as being within the cognitive symptom domain (e.g. "I find it easy to make decisions [reversed item]"). The second factor was defined by seven items and could be interpreted as the affective symptom domain (e.g. "I feel down-hearted and blue"). The third factor was defined by three items and could be interpreted as the somatic symptom domain (e.g. "I eat as much as I used to [reversed item]"). The resulting three-factor structure supports Zung's (1965) original assumption, and replicated previous research (Kitamura et al., 2004; Sakamoto et al., 1998). McDonald's $\omega$ coefficients as measures of internal consistency were acceptable

19 for all three symptom domains (.86 for cognitive, .86 for affective, and .59 for somatic). 
1

from .32 to .56 .

We then compared a first-order three-factor model with higher-order three-factor and bi-factor models. The model fit indices for the confirmatory factor analyses are shown in

Table 2. The results revealed that a bi-factor model explained the data more parsimoniously than the first-order three-factor and higher-order three-factor models. Table 1 also shows

factor loadings for both the general and three specific factors. The standardised loadings on

the general factor ranged from .19 to .76 . This means that the general factor reflects a broad depressive symptomology. Items from the cognitive symptom domain loaded strongly on the general factor and only weakly on the specific factor, indicating that the general factor adequately captured the cognitive dimension. The affective items loaded similarly on the general and the specific factor. Items for the somatic symptom domain loaded more strongly on the specific factor as opposed to the cognitive symptom domain, indicating that the specific factor captured more of the variance in somatic items than the general factor.

\section{Univariate Genetic Analysis}

The heritability of the total sum-score and the sum-scores reflecting the three dimensions are provided in Table 3 . The results from the analyses including opposite sex DZ twins are presented as they were very similar to the results from the analyses excluding opposite sex twins. Estimates indicate that non-additive genetic and shared environmental effects were not detected for any symptom domain and that the AE model fitted the data best, which is consistent with prior empirical studies (e.g. Ball et al., 2009; Chen et al., 
2014, Kendler et al., 2013). The heritability of these symptoms ranged between .27 for the somatic symptom domain and .41 for the cognitive symptom domain.

\section{Multivariate Genetic Analysis}

Given that individual differences between the depressive symptom domains were explained by only additive genetic (A) and non-shared environmental (E) influences, the multivariate genetic analyses utilised in this section implemented an AE model.

Additionally, on the basis of results from the phenotypic confirmatory factor analysis, we selected the best-fitting factorial structure for multivariate genetic modelling, namely a bi-factor solution including a general factor with three specific factors (i.e. cognitive, affective, and somatic symptom domains). As shown in Table 4, the variation in the general factor labelled depression was explained by the common genetic factor (47.0\%), and by the non-shared environmental factor (53.0\%). The symptom domain-specific genetic effect accounted for $29.4 \%$ of the variance only in the affective symptom domain, but no significant amount of variance in the cognitive and somatic symptom domains. The symptom domain-specific nonshared environmental effects, including the measurement error, were significant in all three symptom domains and explained the variance from $69.6 \%$ to $81.8 \%$. In sum, our results revealed a genetic contribution to the general factor of depression, suggesting a set of shared genetic effects across all items. Specific genetic influences were present only for the affective symptom domain. Moreover, in the general factor and even more so in the specific factors, non-shared environmental effects were 
1 prominent.

\section{Discussion}

The aims of this study were: (a) to examine the factorial structure of the SDS in a

4 lesser extent, somatic symptoms. Previous studies have reported that a bi-factor solution

Japanese twin sample; (b) to estimate the relative contributions of genetic and

environmental influences on depressive symptoms dimensions; and (c) to investigate the multivariate genetic and environmental architecture underlying depressive symptom factors.

\section{Factorial structure of depression symptomatology.}

The results of our exploratory factor analysis yielded three factors describing cognitive, affective, and somatic symptomatology. This analysis demonstrated that in a Japanese sample, a three-factor solution describes depressive symptoms better. This is in line with Zung's (1965) original assumption and as reported by previous studies (Kitamura et al., 2004; Sakamoto et al., 1998). Moreover, findings from a series of confirmatory factor analyses supported the idea that a bi-factor model with a general factor and three specific factors fitted the data better than the other alternative models. This suggests that depression may be interpreted as a general psychological construct with three residual factors reflecting cognitive, affective, and somatic symptom domains. This general factor captured the variance in cognitive symptoms more fully, followed by affective symptoms and, to a fits best to the data on other depression scales (e.g. Fong et al., 2016 for the Center for 
1 Epidemiologic Studies Depression Scale, [CES-D], and McElory et al., 2018 for the Beck

2 Depression Inventory II, [BDI-II]). Our study could contribute to the accumulating

3 evidence that, across scales, depression appears more likely to have a bi-factor structure

4 rather than a single factor, multiple factor, or higher-order factor structure.

Within a non-clinical setting, we were able to demonstrate that the structure of depressive symptomatology could be represented by a general factor, which captured individual differences across a broad range of depressive symptoms. In addition to this core general factor, three factors captured the specific variance shared by items within each of

9 three dimensions-affective, cognitive, and somatic. If these findings can be replicated in a clinical sample, this implies that clinicians have to simultaneously attend to depression as a general construct while still attending to specific manifestations, which can be independent of the general construct and of each other. Namely, some patients may manifest somatic symptoms while not having elevated levels of affective and/or cognitive symptoms. As such, differential therapeutic strategies should ideally attend to differential symptomatic profiles.

\section{Genetic and environmental influences on cognitive, affective and somatic}

domains.

Univariate genetic analyses for sum-scores reflecting each dimension were explained by additive genetic and non-shared environmental influences only. These results are 
estimates were rather comparable, ranging from $29 \%$ to $41 \%$ for each of the three symptom domains, and approximately two thirds of the individual differences in each symptom dimension were explained by non-shared environmental influences (Ball et al., 2009, Fernandez-Pujas et al., 2015, Sullivan et al., 2000). Although several previous studies reported significant shared environmental influences (Chen et al., 2014; Happonen et al., 2002), they utilised children and adolescent twin pairs and their parents, whereas the present study was conducted in an adult sample.

\section{Multivariate genetic architecture of depression symptomatology.}

Genetic contributions. We conducted multivariate genetic analyses based on the results of our exploratory and confirmatory factor analyses. In response to the best-fitting model suggested from our factor analyses at the phenotypic level, we fitted a bi-factor model to the data for behavioural genetic modelling. Previous twin research has shown that depression is substantially heritable. However, to the best of our knowledge, this is the first study fitting a bi-factor model within a twin design to examine the aetiology of depression. As shown in Table 1, almost all items loaded substantially on the general factor, suggesting that a common heritable factor may adequately reflect depression symptomatology. Our estimate of the heritability of the general factor of depressive symptoms was substantial (47.0\% [95\% CI: 36.8\%, 57.1\%]), with a higher point estimate than for any of the sum-score reflecting the symptom dimensions. Interestingly, the estimated heritability of the general factor from a bi-factor model was higher than that of the total sum-score. This 
1 suggests that the use of bi-factor architecture reduced the measurement error for the general

2 factor, enabling a better estimation of the aetiological components (Henry et al., 2016).

3 Furthermore, our bi-factor model revealed that a significant specific genetic contribution

4

5 was detected only for the affective symptom domain even though univariate genetic analyses had detected significant heritability for all symptom domains. This suggests that affective symptoms might be influenced by specific genetic influences not captured by the general factor, indicating a partial aetiological independence of the affective symptom domain from the general factor among a Japanese sample.

Overall and in line with the previous studies, our findings supported substantial heritability of the general factor for depressive symptomatology. A shared genetic risk for the general factor of depression may be linked to precursors of depressive symptomatology (e.g. neuroticism of personality traits, Luciano et al., 2018). By contrast, prior research on major depression assessed by personal interview (Kendler et al., 2013) revealed that major depression criteria from the DSM-IV did not reflect a single dimension of genetic liability, indicating that genetic influences on clinical criteria of major depression were heterogeneous and reflected the three underlying dimensions of major depression.

Differences in results could reflect differences in methods, in particular assessment methods and fitted models because Kendler et al. (2013) did not fit a bi-factor model to their data. 
1 previous research demonstrating that depression is moderately heritable (Sullivan et al.,

2 2000). We also found that specific factors were mostly influenced by non-shared

3 environmental rather than genetic influences (except for the affective domain in our

4

5

6

7

8 9 interpretations are plausible. First, the fact that the general factor is more heritable than the

sample). Whether our findings of a bi-factorial structure and the corresponding estimates of

genetic and environmental influences can be replicated using other depression scales

remains to be fully investigated. So far, previous studies have shown that the CES-D and

BDI-II have a bi-factor structure at the phenotypic level (Fong et al., 2016; McElory et al., 2018). Additional research is clearly needed in the area. If our findings are confirmed, two specific factors, which are mainly explained by the E component, may be due to measurement error. The E component includes both genuine non-shared environmental influences and measurement error. Consequently, the specific factors, which by definition rely on less items, may suffer more from measurement error, artificially lowering heritability and increasing non-shared environmental estimates. Alternatively, we can speculate that the general liability to depression is indeed more heritable, whereas specific manifestations of the disease (i.e. cognitive, affective or somatic) are more dependent on person-specific environmental influences. If verified, such a structure might contribute to explain cross-cultural differences in the clinical manifestations of the common liability to depression. 
1 accounted for half of the variance in the general factor, and 70 to $82 \%$ of the variance in the

2 domain-specific factors. Importantly, estimates of non-shared environmental influences

3 also include measurement error. These results raise the possibility that, while a general

4 genetic factor largely accounts for the communality between symptoms of depression,

5 non-shared environmental influences may explain most of the dimension-specific factors.

6 According to prior available research, stressful life situations (e.g. individual loss

7 experience such as a job or a beloved one, and interpersonal conflict at social contexts)

8 could be plausible candidates of non-shared environmental triggers (Mazure, 1998 for a

9 review). Therefore, the utility of such specific factors once the general factor is accounted

10 for should be tested in further studies, for example, by testing whether specific factors

11 uniquely associate with predictors and outcomes of depressive symptomatology.

Strengths and limitations.

The present study has several limitations. One limitation is that because of the

relatively small size of the twin sample, we did not test for sex differences in the measurement and aetiological models. The development of depression may involve sex differences, and females generally do show a higher prevalence of depression. However,

17 previous studies on the heritability of depression showed mixed results: several previous studies reported no differences in heritability between females and males (Agrawal et al.,

19 2004; Chen \& Yu, 2015; Foley et al., 2003), while other studies found that sex differences 
1 behavioural genetic models can help answer the question of whether females and males are

2 influenced by the same amount of genetic and environmental influences, further research

3 with a larger sample size in Asian populations is needed. Another possible limitation is that

4 the twin sample used in the present study is based on a healthy population sample, not a

5 clinical sample. However, Kendler et al. (2006) and Sullivan et al. (2000) have suggested

6 that findings from general population sample studies of depression are comparable to those

7 of clinical sample studies, and research on depression symptomatology in non-clinical

8 samples is important in its own right. Specifically, it can be argued that using a non-clinical

9 sample allowed us to study the phenotypic and genetic architecture of individual

differences in depression using a broad range of depression scores, whereas a clinical

11 sample may entail range restrictions and reduced correlations.

Conclusions.

Despite the above limitations, by using a genetically informative sample, the present

study extended previous findings in several ways. Confirmatory factor analyses at the phenotypic level confirmed the multidimensionality of individual differences in depressive symptoms and, in addition to a general factor, identified three residual factors reflecting three domains of depressive symptoms: cognitive, affective, and somatic. Most importantly, multivariate genetic modelling revealed that the general factor, which adequately reflected depressive symptomatology across dimensions, was moderately heritable, whereas domain specific factors were mostly influenced by non-shared environmental influences. 
3 We are extremely grateful to all the twin families who took part in this study. Financial Support

5 This work was supported in part by the Japan Society for the Promotion of Science

6 KAKENHI Grant-in-Aid for Scientific Research on Innovative Areas (grant no 26118709

7 and $17 \mathrm{H} 05924)$ to $\mathrm{YT}$.

8

\section{Declaration of Interest}

9 None.

\section{Ethical Standards}

11 The authors assert that all procedures contributing to this work comply with the ethical

12 standards of the relevant institutional committees on human experimentation and with the

13 Helsinki Declaration of 1975, as revised in 2008. 


\section{References}

2 Akaike H (1987) Factor analysis and AIC. Psychometrika 52, 317-332.

Agrawal A, Jacobson KC, Gardner CO, Prescott CA, and Kendler KS (2004) A population based twin study of sex differences in depressive symptoms. Twin Research 7, 176-181.

American Psychiatric Association (2013) Diagnostic and Statistical Manual of Mental Disorders, Fifth Edition, Text Revision. Washington, DC, American Psychiatric Association.

Ando J, Fujisawa K, Shikishima C, Hiraishi K, Nozaki M, Yamagata S, Takahashi Y, Ozaki K, Suzuki K, Deno M, Sasaki S, Toda T, Kobayashi K, Sugimoto Y, Okada M, Kijima N, Ono Y, Yoshimura K, Kakihana S, Maekawa H, Kamakura T, Nonaka K, Kato N, and Ooki S (2013) Two cohort and three independent anonymous twin projects at the Keio Twin Research Center (KoTReC). Twin Research and Human Genetics 16, 202-216.

Ball HA, Sumathipala A, Siribaddana SH, Kovas Y, Glozier N, McGuffin P and Hotopf M (2009). Genetic and environmental contributions to depression in Sri Lanka. The British Journal of Psychiatry 195, 504-509.

Byers AL, Levy BR, Kasl SV, Bruce ML, and Allore AG (2009) Heritability of depressive symptoms: A case study using a multilevel approach. International Journal of Methods in Psychiatric Research 18, 287-296. 
1 Chen J, Li X, Natsuaki MN, Leve LD, and Harold GT (2014) Genetic and

2 environmental influences on depressive symptoms in Chinese adolescents. Behavior Genetics 44, 36-44.

Chen J and Yu J (2015) Sex differences in genetic and environmental influences on adolescent depressive symptoms: A meta-analytic review. Depression Research and Treatment Article ID 476238.

Cheung HN and Power MJ (2012) The development of a new multidimensional depression assessment scale: Preliminary results. Clinical Psychology and Psychotherapy 19, 170-178.

Chida F, Okayama A, Nishi N, and Sakai A (2004) Factor analysis of Zung Scale scores in a Japanese general population, Psychiatry and Clinical Neurosciences 58, 420-426.

Falissard B (2012) psy: various procedures used in psychometry. R package version 1.1. Available at: http://cran.r-project.org/package=psy.

Fernandez-Pujals AM, Adams MJ, Thomson P, McKechanie AG, Blackwood DHR, (GS:SFHS). PLoS ONE 10(11): e0142197. 
Major depression and associated impairment: same or different genetic and environmental risk factors? American Journal of Psychiatry 160, 2128-2133.

\section{Fong TCT, Chan CLW, Ho RTH, Chan JSM, Chan CHY, and Ng SM (2016)} Dimensionality of the Center for Epidemiologic Studies Depression Scale: An exploratory bi-factor analytic study. Quality of Life Research 25, 731-737.

Fukuda K and Kobayashi S (1983) Japanese version of Self-rating Depression Scale. Kyoto: Sankyobo.

\section{Happonen M, Pulkkinen L, Kaprio J, Van der Meere J, Viken RJ, and Rose RJ (2002)} The heritability of depressive symptoms: Multiple informants and multiple measures. Journal of Child Psychology and Psychiatry 43, 471-479.

Henry J, Pingault J-B, Boivin M, Rijsdijk F and Viding E (2016) Genetic and environmental aetiology of the dimensions of Callous-Unemotional traits. Psychological Medicine 46, 405-414.

Hofmann RJ (1978) Complexity and simplicity as objective indices descriptive of factor solutions. Multivariate Behavioral Research 13, 247-250.

Holzinger KJ and Swineford F (1937) The bi-factor method. Psychometrika 2, 41-54.

Kawakami N (2007) Epidemiology of depressive disorders in Japan and the world, Nippon Rinsho 65, 1578-1584.

Kendler KS, Aggen SH and Neale MC (2013) Evidence for multiple genetic factors underlying DSM-IV criteria for major depression. JAMA Psychiatry 70, 599-607. 
1 Kendler KS, Gardner CO, Neale MC and Prescott CA (2001) Genetic risk factors for major depression in men and women: similar or different heritabilities and same or partly distinct genes? Psychological Medicine 31, 605-616.

Kendler KS, Gatz M, Gardner CO and Pedersen NL (2006) A Swedish national twin study of lifetime major depression. American Journal of Psychiatry 163, 109-114.

Kessler RC and Bromet EJ (2013) The epidemiology of depression across cultures. Annual Review of Public Health 34, 119-138.

Kitamura T, Hirano H, Chen Z and Hirata M (2004) Factor structure of the Zung Self-rating Depression Scale in first-year university students in Japan. Psychiatry Research 128, 281-287.

Knopik VS, Neiderheiser J, DeFries JC and Plomin R (2016) Behavioral genetics (7th ed.). New York, NY: Worth.

Lee HC, Chiu HF, Wing YK, Leung CM, Kwong PK and Chung DW (1994) The Zung Self-Rating Depression Scale: Screening for depression among the Hong Kong Chinese elderly. Journal of Geriatric Psychiatry and Neurology 7, 216-220.

Luciano M, Hagenaars SP, Davies G, Hill WD, Clarke T-K, Shirali M, Harris SE, Marioni RE, Liewald DC, Fawns-Ritchie C, Adams MJ, Howard DM, Lewis CM, Gale CR, McIntosh AM, and Deary IJ (2018) Association analysis in over 329,000 individuals identifies 116 independent variants influencing neuroticism. Nature Genetics 50, 6-11. 
1 Mazure CM (1998) Life stressors as risk factors in depression. Clinical Psychology:

Science and Practice 5, 291-313.

\section{McElroy E, Casey P, Adamson G, Filippopoulos P and Shevlin M (2018) A} comprehensive analysis of the factor structure of the Beck Depression Inventory-II in a sample of outpatients with adjustment disorder and depressive episode. Irish Journal of Psychological Medicine 35, 53-61.

McGue M and Bouchard TJ Jr. (1984) Adjustment of twin data for the effects of age and sex. Behavior Genetics 14, 325-343.

McGue M and Christensen K (1997) Genetic and environmental contributions to depression symptomatology: Evidence from Danish twins 75 years of age and older. Journal of Abnormal Psychology 106, 439-448.

Magnus P, Berg K and Nance WE (1983) Predicting zygosity in Norwegian twin pairs born 1915-1960. Clinical Genetics 24, 103-112.

Neale MC, Hunter MD, Pritikin JN, Zahery M, Brick TR, Kirkpatrick RM, Estabrook R, Bates TC, Maes HH, and Boker SM (2016) OpenMx 2.0: Extended structural equation and statistical modeling. Psychometrika 81, 535-549.

Olsen JA and Kenny DA (2006) Structural equation modeling with interchangeable dyads. Psychological Methods 11, 127-141.

Ooki S, Yamada K, Asaka A and Hayakawa K (1990) Zygosity diagnosis of twins by questionnaire. Acta Geneticae Medicae et Gemellologiae 39, 109-115. 
Passik SD, Lundberg JC, Rosenfeld B, Kirsh KL, Donaghy K, Theobald D, Lundberg E, and Dugan W (2000) Factor analysis of the Zung Self-Rating Depression Scale in a large ambulatory oncology sample. Psychosomatics 41, 121-127.

\section{Romera I, Delgado-Cohen H, Perez T, Caballero L and Gilaberte I (2008) Factor analysis of the Zung self-rating depression scale in a large sample of patients with major depressive disorder in primary care. BMC Psychiatry 8: 4.}

Rosseel Y (2012) lavaan: An R Package for Structural Equation Modeling. Journal of Statistical Software 48, 1-36.

Sakamoto S, Kijima N, Tomoda A and Kambara M (1998) Factor structures of the Zung Self-Rating Depression Scale (SDS) for undergraduates. Journal of Clinical Psychology 54, 477-487.

Schwarz GE (1978) Estimating the dimension of a model, Annals of Statistics 6, 461-464.

Sclove L (1987) Application of model-selection criteria to some problems in multivariate analysis. Psychometrika 52, 333-343.

Sugawara M, Sakamoto S, Kitamura T, Toda MA and Shima S (1999) Structure of depressive symptoms in pregnancy and the postpartum period. Journal of Affective Disorders 54, 161-169.

Sullivan PF, Neale MC and Kendler KS (2000) Genetic epidemiology of major depression: review and meta-analysis. American Journal of Psychiatry 157, $1552-1562$. 
1 Torgersen S (1979) The determination of twin zygosity by means of a mailed

2 questionnaire. Acta Geneticae Medicae et Gemellologiae 28, 225-236.

3 Wood AC, Rijsdijk F, Saudino KJ, Asherson P and Kuntsi J (2008) High heritability for a composite index of children's activity level measures. Behavior Genetics 38, 266-276

5 World Health Organization (2017) Depression and other common mental disorders:

$6 \quad$ Global health estimates. World Health Organization: Geneva.

7 Zung WWK (1965) A self-rating depression scale. Archives of General Psychiatry 12, $8 \quad 63-70$. 
2 Fig. 1 Three tested models when conducting confirmatory factor analyses.

3 Fig. 2 Scree plotted eigenvalues with simulated random normal data (number of iterations

$4=20)$ 
1 Fig. 1.

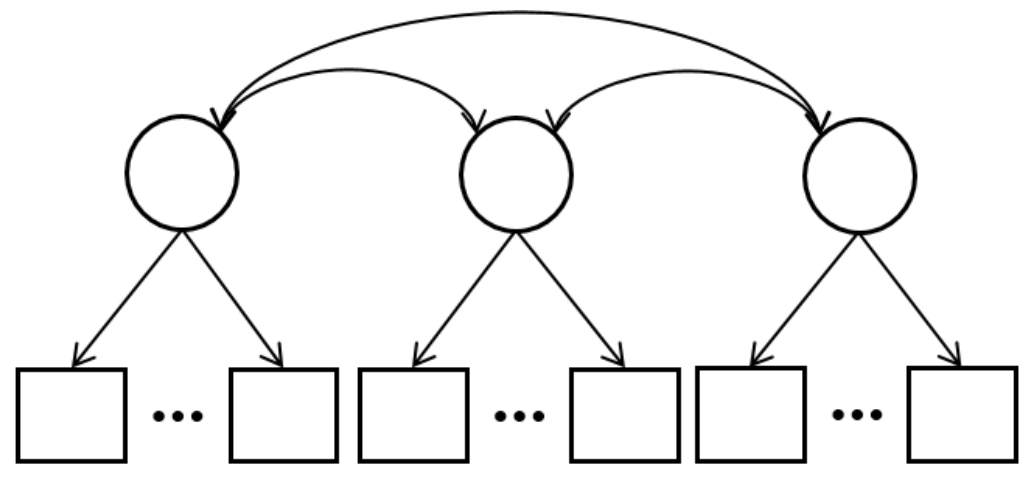

a) simple correlated three factor solution

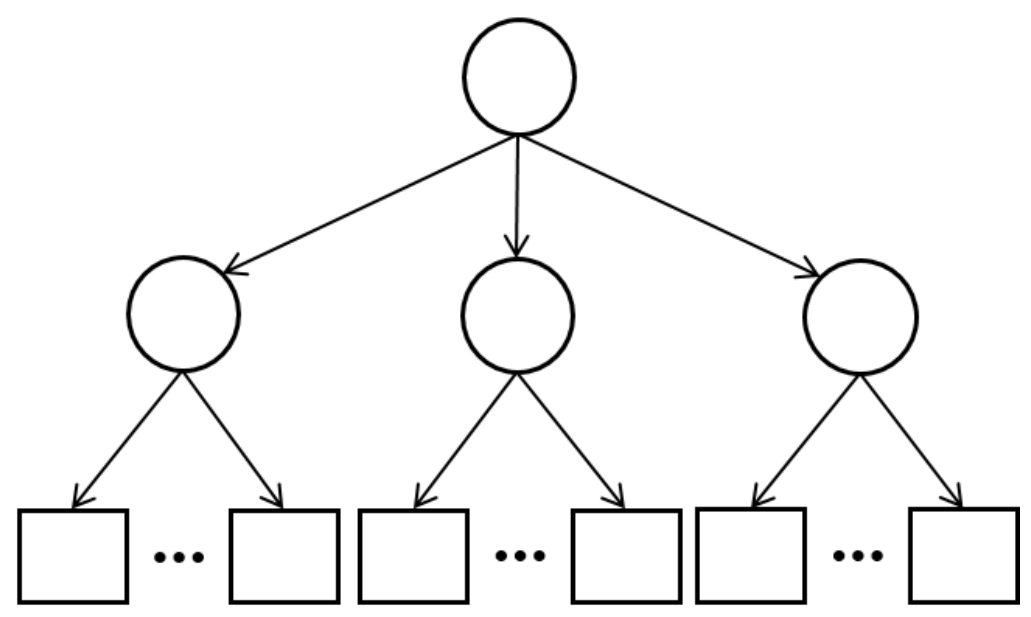

b) higher-order factor solution

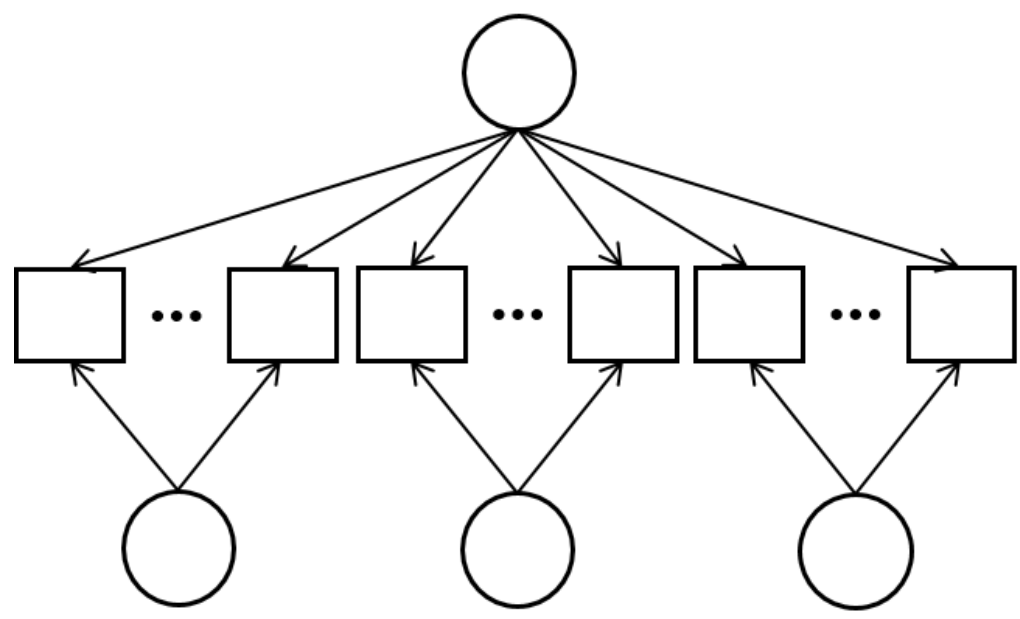

c) bi-factor solution (or general-specific) 
$1 \quad$ Fig. 2.

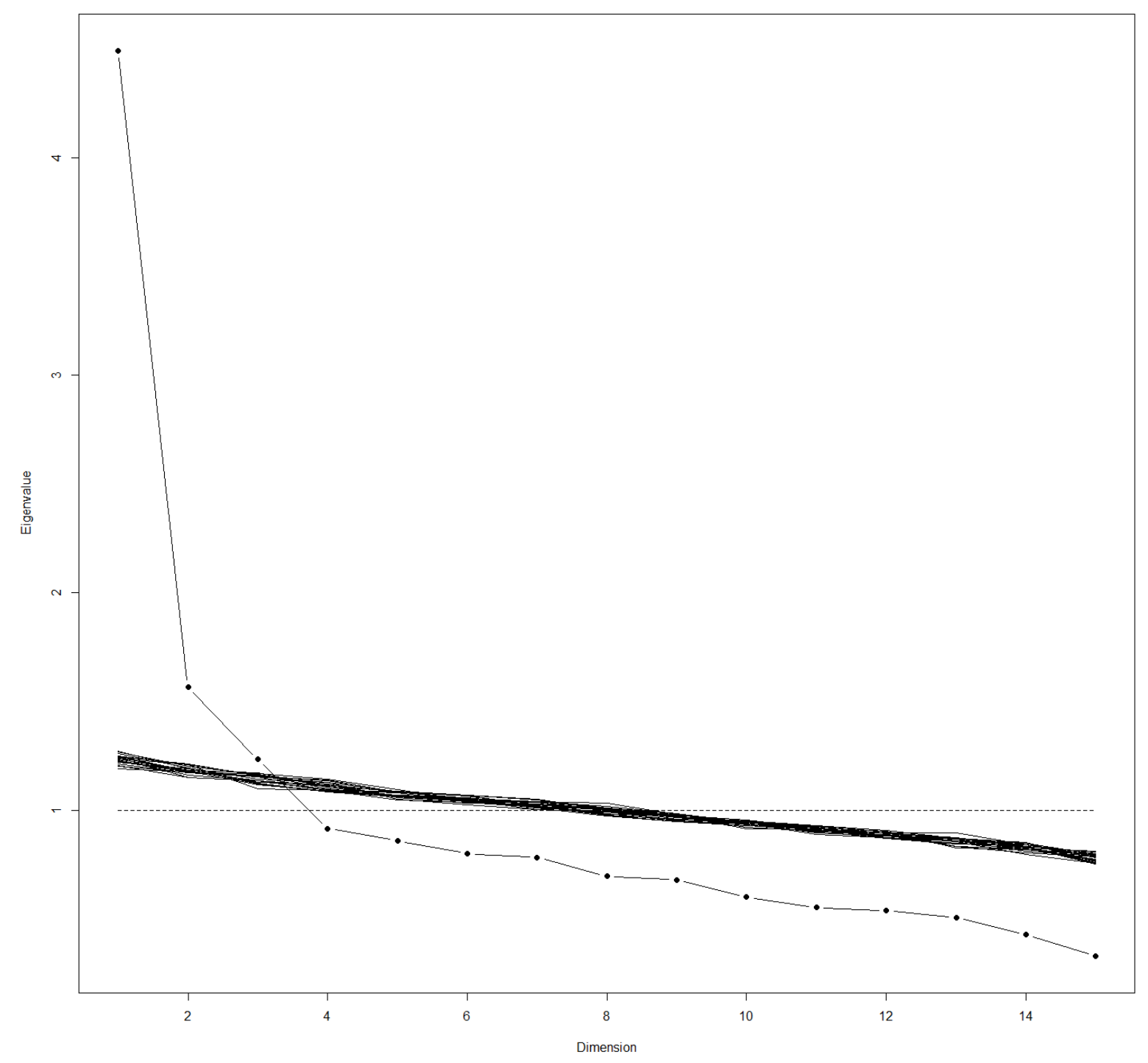


1 Table 1 Exploratory and confirmatory factor analyses for depressive symptoms with

\begin{tabular}{|c|c|c|c|c|c|c|c|c|c|c|}
\hline & \multirow{2}{*}{ Mean } & \multirow{2}{*}{ S.D. } & & \multicolumn{3}{|c|}{ EFA } & \multicolumn{4}{|c|}{$\begin{array}{c}\text { CFA } \\
\text { with a bi-factor solution }\end{array}$} \\
\hline & & & & $\mathrm{F} 1$ & $\mathrm{~F} 2$ & F3 & G & $\mathrm{C}$ & A & S \\
\hline \multicolumn{11}{|l|}{ F1: cognitive symptom domain $(\omega=.86)$} \\
\hline I still enjoy the things I used to do (R) & 2.35 & .90 & & .74 & .01 & .03 & .76 & .62 & & \\
\hline My life is pretty full (R) & 2.26 & .91 & & .74 & .05 & .03 & .74 & .16 & & \\
\hline I feel hopeful about the future (R) & 2.23 & 1.01 & & .62 & -.04 & .02 & .65 & -.10 & & \\
\hline I feel that I am useful and needed (R) & 2.32 & .92 & & .60 & -.01 & -.04 & .62 & -.13 & & \\
\hline I find it easy to make decisions (R) & 2.69 & .89 & & .41 & .07 & -.07 & .49 & -.15 & & \\
\hline \multicolumn{11}{|l|}{ F2: affective symptom domain $(\omega=.86)$} \\
\hline I have crying spells or feel like it & 2.02 & 1.00 & & -.10 & .67 & .00 & .30 & . & .55 & \\
\hline I am more irritable than usual & 1.91 & .93 & & .01 & .65 & .03 & .40 & & .55 & \\
\hline I feel down-hearted and blue & 2.16 & .98 & & .18 & .65 & -.01 & .54 & & .51 & \\
\hline I get tired for no reason & 2.79 & .97 & & .00 & .49 & .04 & .32 & & .40 & \\
\hline My mind is as clear as it used to be (R) & 2.53 & .90 & & .28 & .46 & -.05 & .53 & & .34 & \\
\hline My heart beats faster than usual & 1.57 & .82 & & -.07 & .45 & .14 & .25 & & .40 & \\
\hline I am restless and can't keep still & 1.90 & .93 & & -.07 & .44 & .12 & .24 & & .39 & \\
\hline \multicolumn{11}{|l|}{ F3: somatic symptom domain $(\omega=.59)$} \\
\hline I eat as much as I used to $(\mathrm{R})$ & 1.39 & .69 & & .03 & .04 & .49 & .23 & & & .53 \\
\hline I have trouble sleeping at night & 1.93 & 1.06 & & .03 & .13 & .47 & .28 & & & .39 \\
\hline I still enjoy sex $(\mathrm{R})$ & 1.94 & .90 & & .17 & -.19 & .35 & .19 & & & .30 \\
\hline \multicolumn{11}{|l|}{ Removed } \\
\hline Morning is when I feel the best (R) & 3.06 & .87 & & & & & & & & \\
\hline I notice that I am losing weight & 1.76 & .93 & & & & & & & & \\
\hline I have trouble with constipation & 1.93 & 1.09 & & & & & & & & \\
\hline I find it easy to do the things I used to (R) & 1.93 & .86 & & & & & & & & \\
\hline \multirow[t]{2}{*}{ I feel that others would be better off if I were dead } & 1.46 & .76 & & & & & & & & \\
\hline & & & F1 & - & & & & & & \\
\hline \multirow[t]{2}{*}{ inter-factor correlations } & & & $\mathrm{F} 2$ & .56 & - & & & & & \\
\hline & & & F3 & .37 & .32 & - & & & & \\
\hline
\end{tabular}
Note. R: reversed items. $\omega$ : McDonald's omega coefficients. EFA: Exploratory Factor

5 Analysis. CFA: Confirmatory Factor Analysis. Standardized factor loadings are presented

6 for EFA and CFA. The CFA model presented here is a bi-factor solution, with G, C, A, S

7 standing for the general, cognitive, affective, and somatic factors respectively. Values in the

8 bottom three rows indicate inter-factor correlations when applying exploratory factor

9 analysis. 
1 Table 2 Model fit indices to compare four confirmatory factor analyses

\begin{tabular}{lcccc}
\hline & AIC & $\begin{array}{c}\text { sample-size } \\
\text { adjusted BIC }\end{array}$ & RMSEA & SRMR \\
\hline a) three-factor solution & 30673.744 & 30741.367 & .035 & .049 \\
b) higher-order factor solution & 30674.509 & 30733.462 & .035 & .052 \\
c) bi-factor solution & $\mathbf{3 0 6 3 1 . 8 4 0}$ & $\mathbf{3 0 7 1 4 . 2 0 0}$ & $\mathbf{. 0 3 3}$ & $\mathbf{. 0 4 5}$ \\
\hline
\end{tabular}

2

3 Note: AIC: Akaike's Information Criteria, BIC: Bayesian Information Criteria, RMSEA:

4 Root Mean Square Error of Approximation, SRMR: Standardized Root Mean Square

$5 \quad$ Residual. Bold face indicates the best fitting model. 
1 Table 3 Genetic and environmental influences on sum-scores indicating three symptom

2 domains and a total score for depression

\begin{tabular}{|c|c|c|c|c|c|c|c|c|c|}
\hline & Models & $a^{2}$ & $d^{2}$ & $c^{2}$ & $e^{2}$ & $-2 \mathrm{LL}$ & $d f$ & AIC & BIC \\
\hline \multirow[t]{5}{*}{$\begin{array}{c}\text { cognitive } \\
\text { symptom domain }\end{array}$} & $\mathrm{ADE}$ & $\begin{array}{c}.30 \\
(.00-.49)\end{array}$ & $\begin{array}{c}.11 \\
(.00-.50)\end{array}$ & - & $\begin{array}{c}.59 \\
(.50-.69)\end{array}$ & 4475.427 & 843 & 2789.427 & -626.485 \\
\hline & ACE & $\begin{array}{c}.41 \\
(.12-.49)\end{array}$ & - & $\begin{array}{c}.00 \\
(.00-.00)\end{array}$ & $\begin{array}{c}.59 \\
(.51-.69)\end{array}$ & 4475.558 & 843 & 2789.558 & -626.353 \\
\hline & $\mathbf{A E}$ & $\begin{array}{c}.41 \\
(.31-.49)\end{array}$ & - & - & $\begin{array}{c}.59 \\
(.51-.69)\end{array}$ & 4475.558 & 844 & 2787.558 & -632.405 \\
\hline & $\mathrm{CE}$ & - & - & $\begin{array}{c}.32 \\
(.23-.40)\end{array}$ & $\begin{array}{c}.68 \\
(.60-.77)\end{array}$ & 4482.712 & 844 & 2794.712 & -625.251 \\
\hline & E & - & - & - & 1 & 4528.903 & 845 & 2838.903 & -585.112 \\
\hline \multirow[t]{5}{*}{$\begin{array}{c}\text { affective } \\
\text { symptom domain }\end{array}$} & $\mathrm{ADE}$ & $\begin{array}{c}.20 \\
(.00-.40)\end{array}$ & $\begin{array}{c}.11 \\
(.00-.41)\end{array}$ & - & $\begin{array}{c}.69 \\
(.59-.79)\end{array}$ & 4821.951 & 843 & 3135.951 & -279.961 \\
\hline & ACE & $\begin{array}{c}.31 \\
(.00-.41)\end{array}$ & - & $\begin{array}{c}.00 \\
(.00-.28)\end{array}$ & $\begin{array}{c}.69 \\
(.59-.79)\end{array}$ & 4822.055 & 843 & 3136.055 & -279.856 \\
\hline & $\mathbf{A E}$ & $\begin{array}{c}.31 \\
(.21-.41)\end{array}$ & - & - & $\begin{array}{c}.69 \\
(.59-.79)\end{array}$ & 4822.055 & 844 & 3134.055 & -285.908 \\
\hline & $\mathrm{CE}$ & - & - & $\begin{array}{c}.26 \\
(.16-.34)\end{array}$ & $\begin{array}{c}.74 \\
(.66-.84)\end{array}$ & 4825.644 & 844 & 3137.644 & -282.320 \\
\hline & E & - & - & - & 1 & 4854.198 & 845 & 3164.198 & -259.817 \\
\hline \multirow[t]{5}{*}{$\begin{array}{c}\text { somatic } \\
\text { symptom domain }\end{array}$} & $\mathrm{ADE}$ & $\begin{array}{c}.18 \\
(.00-.38)\end{array}$ & $\begin{array}{c}.12 \\
(.00-.39)\end{array}$ & - & $\begin{array}{c}.70 \\
(.61-.81)\end{array}$ & 3401.399 & 842 & 1717.399 & -1694.460 \\
\hline & ACE & $\begin{array}{c}.29 \\
(.00-.39)\end{array}$ & - & $\begin{array}{c}.00 \\
(.00-.29)\end{array}$ & $\begin{array}{c}.71 \\
(.61-.81)\end{array}$ & 3401.495 & 842 & 1717.495 & -1694.365 \\
\hline & $\mathbf{A E}$ & $\begin{array}{c}.29 \\
(.19-.39)\end{array}$ & - & - & $\begin{array}{c}.71 \\
(.61-.81)\end{array}$ & 3401.495 & 843 & 1715.495 & -1700.417 \\
\hline & $\mathrm{CE}$ & - & - & $\begin{array}{c}.25 \\
(.16-.34)\end{array}$ & $\begin{array}{c}.75 \\
(.66-.84)\end{array}$ & 3404.381 & 843 & 1718.381 & -1697.530 \\
\hline & E & - & - & - & 1 & 3431.302 & 844 & 1743.302 & -1676.661 \\
\hline \multirow[t]{5}{*}{$\begin{array}{l}\text { total depression } \\
\text { score }\end{array}$} & $\mathrm{ADE}$ & $\begin{array}{c}.21 \\
(.00-.48)\end{array}$ & $\begin{array}{c}.19 \\
(.00-.49)\end{array}$ & - & $\begin{array}{c}.60 \\
(.51-.71)\end{array}$ & 5763.317 & 840 & 4083.317 & 679.562 \\
\hline & $\mathrm{ACE}$ & $\begin{array}{c}.39 \\
(.12-.48)\end{array}$ & - & $\begin{array}{c}.00 \\
(.00-.00)\end{array}$ & $\begin{array}{c}.61 \\
(.52-.71)\end{array}$ & 5763.657 & 840 & 4083.657 & 679.902 \\
\hline & $\mathbf{A E}$ & $\begin{array}{c}.39 \\
(.29-.48)\end{array}$ & - & - & $\begin{array}{c}.61 \\
(.52-.71)\end{array}$ & 5763.657 & 841 & 4081.657 & 673.850 \\
\hline & $\mathrm{CE}$ & - & - & $\begin{array}{c}.31 \\
(.22-.39)\end{array}$ & $\begin{array}{c}.69 \\
(.61-.78)\end{array}$ & 5770.963 & 841 & 4088.963 & 681.156 \\
\hline & E & - & - & - & 1 & 5812.797 & 842 & 4128.797 & 716.938 \\
\hline
\end{tabular}

4 Note. Numbers in parentheses show $95 \%$ confidence intervals. $a^{2}=$ additive genetic effect,

$5 \quad d^{2}=$ non-additive genetic effect, $c^{2}=$ shared environmental effect, $e^{2}=$ non-shared

6 environmental effect. $-2 \mathrm{LL}=-2 \times \log$ Likelihood, $-2 \mathrm{LL}$ follows a $\chi^{2} d f$ distribution, $d f=$ 
1 degree of freedom, AIC $=$ Akaike's information criteria, $\mathrm{BIC}=$ Bayesian information 2 criteria. Bold faces indicate best fitting models. 
1 Table 4 Genetic and non-shared environmental parameters on each factor from the bi-factor 2 solution.

3

\begin{tabular}{lcc}
\hline & $a^{2}$ & $e^{2}$ \\
\hline \multirow{2}{*}{ General factor of depression } & .470 & .530 \\
Cognitive-specific symptom domain & {$[.368, .571]$} & {$[.429, .632]$} \\
& .182 & .818 \\
Affective-specific symptom domain & {$[.000, .515]$} & {$[.485,1.000]$} \\
\multirow{2}{*}{ Somatic-specific symptom domain } & .294 & .706 \\
& {$[.145, .471]$} & {$[.529, .855]$} \\
\end{tabular}

4 